\title{
The algorithm for finding potentially oscillating behavior in enzymatic systems
}

\author{
Tatiana N. Lakhova \\ Sector of Bioinformatics \\ and Information \\ Technologies in Genetics \\ Kurchatov genomics center \\ ICG SB RAS \\ Novosibirsk, Russia \\ tlakhova@bionet.nsc.ru
}

\author{
Fedor V. Kazantsev \\ Sector for computer \\ analysis and simulations of \\ biological systems \\ Kurchatov genomics center \\ ICG SB RAS \\ Novosibirsk, Russia \\ kazfdr@bionet.nsc.ru
}

\author{
Yuriy G. Matushkin \\ Laboratory of molecular \\ genetic systems \\ ICG SB RAS \\ Novosibirsk, Russia \\ mat@bionet.nsc.ru
}

\author{
Sergey A. Lashin \\ Sector of Bioinformatics \\ and Information \\ Technologies in Genetics \\ Kurchatov genomics center \\ ICG SB RAS \\ Novosibirsk State \\ University \\ Novosibirsk, Russia \\ lashin@bionet.nsc.ru
}

\begin{abstract}
Enzymatic reactions regulate a lot of processes in a cell. Chemical compounds such as various inhibitors, activators, cofactors, allosteric regulators, etc. may influence reaction rate. We are interested in the oscillatory processes that play role in the functioning of biological systems. It is known that oscillatory behavior frequently emerges via influence of the positive and/or negative feedback loops. Development and analysis of mathematical and computer models may be the tools to explore such behavior. Therefore, the aim of our research is to develop the technology that can help find potentially oscillating microbial enzymatic subsystems and to explore their behavior.
\end{abstract}

Keywords - mathematical model, feedback loop, oscillatory behavior, microbial enzymatic system

\section{Motivation and Aim}

Enzymatic reactions play an important role in metabolic activity of microbial cells. Series of reactions combines into the chain of substance modification, forming metabolic pathways. Often, enzymatic reaction products both may activation and slow down the reaction rate not only self but processes before or after it on the metabolic pathway. This influence is called positive or negative feedbacks. These feedbacks are responsible for complex behavior of biological systems functioning such as oscillations, switches etc.

Exploring of this complex metabolic network behavior frequently requires mathematical and computer modeling approaches.

In this study, we are focused on the processes with oscillatory behavior. It is unclear how to highlight such processes in a metabolic network; what properties identify the oscillatory behavior in the cells as well as if some nonoscillatory systems may become oscillatory under certain conditions or not.

Network reaction properties can only be fully understood by viewing experimental data from a theoretical perspective and by quantitative mathematical modelling of such processes. So, the aim of our research is to develop the technology that can highlight potentially oscillating microbial enzymatic reaction chains and to explore their behavior.

\section{Results}

We have developed the pipeline that can help find potentially oscillating enzymatic subsystems in metabolic network and to explore their behavior. The algorithm consist of the following steps:

The input of the algorithm receives structural (graph) model of a metabolic network.

Structural model decomposition (i.e. finding of subgraphs) and processing analysis have performed with Python to get network contours that are fits to certain criterion. Visual analysis of the selected contours has been carried out with Cytoscape (https://cytoscape.org/).

For each contour, we compiled complete mathematical model in terms of ordinary differential equations. We have used the Mammoth database (http://mammoth.biomodelsgroup.ru) [1] on this step as source of adapted to experimental data set of Escherichia coli enzymatic reaction mathematical models (we processed over 300 enzymatic reaction models).

Computer simulations were performed with Copasi (http://copasi.org). On this step, we have selected the parameters of models to find oscillatory behavior.

At the end, we have found several directed circuits in the structural model each of which has been checked for potential oscillation behavior. We have shown oscillatory behavior of the directed circuits involved into the tryptophan synthesis pathway.

There are a lot of open access databases containing information on metabolic pathways and gene networks that could be used as data source for model reconstruction process.

\section{ACKNOWLEDGMENT}

The study was supported by the Budget Project No. 0324-2019-0040-C-01 and Kurchatov Genomics Center of ICG SB RAS.

\section{REFERENCES}

[1] Kazantsev F, Akberdin I, Lashin S, Ree N, Timonov V, Ratushny A, et al. MAMMOTh: A new database for curated mathematical models of biomolecular systems. J Bioinform Comput Biol. 2018;16:1740010 (16 pages). doi:10.1142/S0219720017400108. 